\title{
Cracking the Code on Quality and Safety in Healthcare - What Will It Take?
}

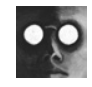 \\ INTRODUCTION \\ Chris Power, MHSC \\ CEO \\ Canadian Patient Safety Institute (CPSI) \\ Edmonton, $\mathrm{AB}$
}

Over the next 30 years in Canada, within acute and home care settings, there could be roughly 400,000 average annual cases of patient safety incidents (PSIs), costing approximately $\$ 6,800$ per patient and generating an additional $\$ 2.75$ billion in healthcare treatment costs per year, according to a recent report by RiskAnalytica (2017). The PSIs considered and the costs incurred are all preventable! This is equivalent to such events occurring in Canada every 1 minute and 18 seconds, and a resulting death every 13 minutes and 14 seconds (RiskAnalytica 2017). No other industry would tolerate those statistics, so why do they continue to exist in such large numbers in healthcare? What will it take to "crack the code" on creating safe environments and practice in healthcare?
What the global patient safety experts tell us is that there is no simple solution to reducing preventable patient safety incidents but rather a combination of many initiatives as well as standardization, regulation and policy. Having led safety improvement collaboratives at the front line for many years, CPSI has found that this was incredibly important work but simply not enough on its own to effect the kind of change that is required. Initiatives focused on creating a culture of safety, teamwork, communication and the importance of visible leadership at all levels of the organization are essential if we are to "crack the code."

Gardam, et al. (2017) have put forth an approach to staff-led quality improvement, front-line ownership (FLO), that is demonstrating increased engagement and 
improvement in specific areas. Although not yet evaluated and highly customized to the local context, FLO speaks to the importance of engaging some of the players who will be impacted by the improvement being tested. The respondents to the lead paper (Bailey and Bevan 2017; Davies 2017; Hughes et al. 2017; Plsek 2017; Robson 2017; Thompson and Clement 2017) have all seen value in the approach. Yet, a number of themes have emerged from their commentaries that speak to additional elements required to achieve a culture of safety and quality and move the bar on our abysmal statistics, such as:

- The importance of engaging and partnering with patients.

- Evaluation so that we can contribute to the evidence to demonstrate what works.

- Leadership.

- Sustainability.

- Standardization vs customization and the role both play in creating safe environments.

As the only national organization dedicated to patient safety, CPSI has developed a new strategy for the next five years that will propel Canada towards having the safest healthcare in the world. Patient Safety Right Now - not in 6 months or 6 years - but RIGHT NOW, is what we are calling this new strategy. We believe that we need to draw a line in the sand and say enough is enough. It can no longer be acceptable to opt out of evidence-based, proven best practices that are known to reduce or eliminate harm. And although I fully understand the need of physicians and staff to feel that they are determining areas for improvement that are important to them, there are some nonnegotiables that leaders must implement and enforce so that practice is improved and patient safety is achieved. As has been pointed out in the papers, it is not one versus the other.
Every person working in healthcare

... has a responsibility to contribute

to improving the quality and safety

of care in this country.

Standardization is incredibly important for those processes that are evidence-based and the local context is always a necessary consideration when determining how to implement improvement projects. What we have fallen short on in the quality and safety world despite amazing efforts on the part of so many - is truly demonstrating what works, spreading and scaling it and then sustaining the improvement. CPSI's new strategy will be focused on demonstrating what works through collaboratives based on best practice, evaluating the work and then spreading and scaling the safety improvement to create sustainable change. The other direction we will take is to strengthen commitment to patient safety through awareness, education, transparent reporting, standardization, regulation and policy levers. Every person working in healthcare, whether at the practice, research, leadership or policy level, has a responsibility to contribute to improving the quality and safety of care in this country.

There are many tools needed in our patient safety tool belt but perhaps one of the most important is how we ensure that patients and families are true partners in their care. I was delighted to see that every one of the respondents commented on the necessity of engaging patients. At CPSI, through one of our programs, "Patients for Patient Safety Canada," patients are engaged in all of our work and co-design every resource that is developed. It is often difficult for providers to know how or where to begin to partner with patients on quality and safety initiatives but it is an essential component if we are to reduce the rates of harm. Nothing About Us Without Us 
is meant for every person who delivers or receives care and I would encourage those engaged in FLO work to have patients with you on every step of this journey, including determining the areas that most need improvement.

At a recent symposium that I attended, the importance of patient stories was discussed.

The impact of often heart-wrenching stories of harm was being espoused as a powerful tool to help people put a face to the harm that has been experienced by patients and their families as well as care providers. While most in the room agreed that stories were excellent methods of teaching and learning, there were a few dissenters who said that they did not diminish the value of stories but they preferred to understand the facts rather than the emotions. We know that we need to appeal to both the heart and the head so various approaches are required to create the conditions for staff to be invested in improvement work. FLO is but one approach that shows promise. Evaluating this work will be an important next step in gathering the evidence of demonstrating what works.

For preventable harm to occur every 1 minute and 18 seconds is unacceptable at any level. Together, we can and must improve. Those we have the privilege to serve deserve no less. Patient Safety Right Now!

\section{References}

Bailey, S. and H. Bevan. 2017. "Quality Improvement: Lessons from the English National Health Services." Healthcare Papers 17(1): 49-55. doi:10.12927/ hсpap.2017.25333.

Davies, M. 2017. “A Matter of Balance: Sharing Front-Line Ownership for Quality and Safety with Patients and Families - Scaling and Spreading Local Improvements for Broader Impact." Healthcare Papers 17(1): 44-48. doi:10.12927/ hсpap.2017.25334.

Gardam, M., L. Gitterman, L. Rykert, E. Vicencio and E. Bailey. 2017. "Five Years of Experience Using Front-Line Ownership to Improve Healthcare Quality and Safety." Healthcare Papers 17(1): 8-23. doi:10.12927/hcpap.2017.25339.

Hughes, L., K.R. Busija, E.R. Chaudhuri and I.C. Popescu. 2017. "Patients as Front-Line Owners and Partners in Improving Quality and Safety." Healthcare Papers 17(1): 25-28. doi:10.12927/ hсpap.2017.25338.

Plsek, P. 2017. "Understanding the System You Are in Is Key to Improving It." Healthcare Papers 17(1): 38-43. doi:10.12927/hcpap.2017.25335.

RiskAnalytica. 2017. The Case for Investing in Patient Safety. Toronto, ON: Author.

Robson, B. 2017. "Front-Line Ownership - Necessary but not Sufficient." Healthcare Papers 17(1): 34-37. doi:10.12927/hcpap.2017.25336.

Thompson, L.J. and L. Clement. 2017. "People Powered Health ${ }^{\mathrm{TM}}$ : Taking Front-Line Ownership to the Next Level." Healthcare Papers 17(1): 29-33. doi:10.12927/hcpap.2017.25337.

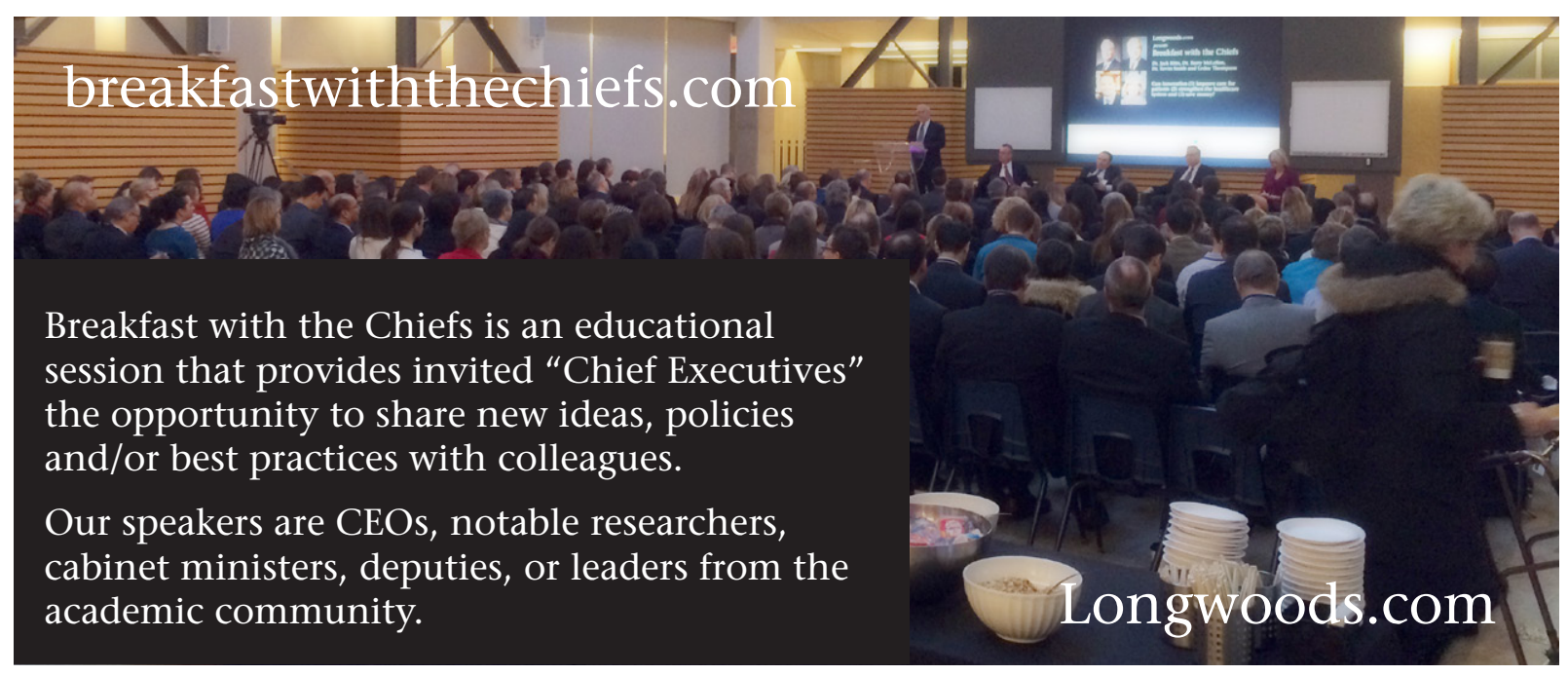

